\title{
The Bioelectricity Revolution: A Discussion Among the Founding Associate Editors
}

\author{
Moderator: Dany Spencer Adams, Editor-in-Chief \\ Associate Editors, in order of appearance: Mustafa Djamgoz, Michael Levin, Min Zhao, \\ Ann Rajnicek, Richard Nuccitelli, Annarosa Arcangeli, Richard Kramer, and Emily Anne Bates ${ }^{9}$
}

\section{Meet the Associate Editors}

Dany Spencer Adams: Thank you all for joining me to discuss Bioelectricity, the journal and the field. Let's start with introductions. Mustafa, would you like to begin?

Mustafa Djamgoz: Yes, of course. I am Mustafa Djamgoz, Professor of Cancer Biology at Imperial College London. Before that, I was a professor of neurobiology. I think it is my rather unusual position in bioelectricity, having held two professorships and sort of catalyzed the neuroscience and the oncology fields through ion channels, to have opened more or less a new field of investigation, the bioelectricity of cancer, obviously, with all my colleagues, including, especially, Dr. Mike Levin.

Being involved in the field of bioelectricity has been an absolutely fascinating journey for me, seeing cancer cells generate the kinds of electrical signals that one might only expect to see in neurons and muscles, the so-called excitable cells. I think the whole notion of excitability needs to be defined. It is one of the things I would like to do in the journal.

And, this has not only just been a nice journey, it has now led to real clinical potential, so we are planning a first clinical trial of metastatic disease focused upon an ion channel blocker, which is very exciting.

DSA: Wow, congratulations. Thank you. Mike, would you like to go next?

Michael Levin: Sure. I am Mike Levin. I am a Professor of Biology at Tufts University, and my original background was actually computer science. I was a software engineer interested in artificial intelligence and robotics. I fell in love with developmental biology and embryos as a premier example of how complex systems can self-assemble, self-repair, and behave adaptively. It led me to study how living tissues store and process information; it seemed to me that bioelectrical signaling would be an ideal way for cells to communicate and compute as they make decisions about what to build or remodel.

Around '97, '98 or so, I started working on creating functional molecular tools to probe long-range and local bioelectric signaling in embryos, in particular at that time in chick and frog embryos, as they tried to sort out which side was left and which side was going to be right.

Since then we have been working on many aspects of bioelectricity as it relates to everything from cancer, to embryonic patterning, adult organ regeneration, synthetic biology, and so on.

\section{DSA: Excellent. Thank you. Min, would you like to share your experience?}

Min Zhao: Hi. My name is Min Zhao, and I am currently a Professor in the Department of Dermatology and Department of Ophthalmology at the University of California [UC], Davis.

I was trained as a trauma surgeon in China and went to London to work with Geoff Burnstock on purinergic receptors. In '94 I joined Colin McCaig and John Forrester to start working on this fascinating question: how do wounds use electric signaling to heal? That brought me into this field.

I am still fascinated by this wonderful phenomenon, to see that the tissue generates its own electricity, as Mustafa just said, outside of the neurons and muscles, in the nonexcitable cells, like the epithelial cell, the fibroblasts, and other types of cells, which we normally do not think of as electrically active at the tissue level. So we are trying to understand these things in the wound healing context and trying to see whether we can develop therapeutic strategies. We are still working on it.

${ }^{1}$ Department of Biology, Tufts University, Medford, Massachusetts.

${ }^{2}$ Department of Biological Sciences, Neuroscience Solutions to Cancer Research Group, Imperial College London, London, England.

${ }^{3}$ Allen Discovery Center at Tufts University, Department of Biology, Medford, Massachusetts.

${ }_{5}^{4}$ Department of Dermatology, University of California Davis, Hayward, California.

${ }^{5}$ Institute of Medical Sciences, University of Aberdeen, Aberdeen, Scotland.

${ }^{6}$ Research and Development, Pulse Biosciences, Davis, California.

${ }^{7}$ Dipartimento di Medicina Sperimentale e Clinica, Universitá degli Studi di Firenze, Florence, Italy.

${ }^{8}$ Helen Wills Neuroscience Institute, University of California, Berkeley, California.

${ }^{9}$ Section of Developmental Biology, Department of Pediatrics, University of Colorado, Denver, Colorado. 
DSA: It is fascinating, isn't it?

MZ: Yes. Very interesting, indeed.

DSA: Ann, can you tell us what captured your imagination and what you do?

Ann Rajnicek: Sure. I am Ann Rajnicek. I work at the University of Aberdeen in Scotland. And I have been interested in bioelectricity ever since I started my $\mathrm{PhD}$. I was doing my research rotations, deciding what lab to work at at Purdue University, and I ended up working in the lab with Ken Robinson, and I was absolutely smitten and enthused with the idea that this simple electrical cue could be so important for so many things.

And I have since that time been working on issues related to wound healing, wound repair, and tissue regeneration, with particular interest and emphasis on central nervous system regeneration, and more recently I've been looking into immune cells and how immune cell function can be tweaked by bioelectric signals.

I am excited to be part of this project, and I was absolutely delighted when Dany approached me about being one of the associate editors of the journal, because I think it is a really exciting time for bioelectricity right now. There are so many new things, and as you will hear from the people that are talking with us today, there are lots and lots of different ideas and potentials that we can explore.

DSA: I agree. Thanks, Ann. Richard Nuccitelli, could you kindly introduce yourself?

Richard Nuccitelli: Sure, I am Rich Nuccitelli, currently the Chief Science Officer at Pulse Biosciences. Our company is commercializing the use of nanosecond pulsed electric fields to treat tumors and skin lesions. I have worked in the field of Bioelectricity my entire career, beginning with the invention of the self-referencing vibrating probe with Lionel Jaffe. This instrument has been used by dozens of investigators over the past 30 years to detect the ionic currents flowing through nearly all cells and embryos due to the polarized distribution of ion channels and pumps. I was a professor at UC Davis for 23 years, where I studied the galvanotaxis of fibroblasts and keratinocytes as well as the mechanism leading to the sperminduced calcium wave in the frog egg. Following that I was a professor for 3 years at the Frank Reidy Research Center for Bioelectrics at Old Dominion University, where I learned about the amazing ability of nanosecond pulses to penetrate into cells and tissues and trigger immunogenic cell death. My wife and I then started BioElectroMed Corp, which has now become Pulse Biosciences.

Bioelectricity is fundamental to all of life. Not only do we generate all of our ATP [adenosine triphosphate] using the electromotive force of hundreds of millivolts in mitochondria, but also all of our senses rely on the flow of ionic currents. From the dark current in retinal rods to the mechanical gating of channels in the hair cells in our ears, we see, hear, taste, and smell with bioelectricity.

DSA: Annarosa Arcangeli, would you introduce yourself?

Annarosa Arcangeli: I am Annarosa Arcangeli, Professor of General Pathology at the University of Florence. I have an
MD, with a specialization in Hematology and Oncology, followed by a PhD in Experimental Oncology. I have been studying cancer biology, and in particular cancer metabolism, since the beginning of my scientific career, in the ' 80 s. During my first studies I jumped suddenly in the bioelectricity field, studying the role of ion fluxes in leukemia differentiation. Afterward, thanks to a stage in Prof. Wanke's laboratory in Milan, I met the electrophysiology world, being immediately fascinated by ion currents, which I studied in excitable (neurons) and not excitable (erythroleukemia) cells.

I have worked with colleagues present around this table to discover the unexpected role of ion channels in cancer cells, and more recently, to define the conductive and not conductive role of these fascinating membrane proteins.

I want to stress here that impressive achievements in the treatment of cancer patients have been obtained in the last twenty years. Nevertheless, cancer is still a devastating disease, let me say for both patients and her/his relatives. Therefore, novel insights in the understanding of the biology of cancer, including its electrical bases, focused to define novel therapeutic strategies, are mandatory.

DSA: Absolutely. Thank you. Richard Kramer, would you tell us about yourself next?

Richard Kramer: Sure. Yes. This is Rich Kramer. I am a professor at UC Berkeley, Molecular Cell Biology Department. I have worked for many years on various aspects of electrophysiology in all kinds of cells, from ion channel structure function to excitability to synaptic transmission, mainly in the nervous system. But I have been interested in other things as well.

Most recently, we have been developing and using tools to impart light sensitivity on different kinds of ion channels and receptors. This is a bit different than what might be called "conventional" optogenetics, which uses exogenous expression of microbial opsins for photocontrol. Instead, we use synthetic chemistry to plug photoisomerizable molecules onto endogenous ion channels or receptors, and control these with light. We call this optopharmacology, in contrast to optogenetics.

One of the places we are using this approach is in the eye, where optopharmacology may be useful for restoring light sensitivity in blinding diseases like retinitis pigmentosa, an inherited disorder where rods and cones degenerate. We are interested in the basic science of the retina, including understanding plasticity associated with photoreceptor loss as well as the therapeutic development of these compounds as potential treatments for blindness in humans.

DSA: I have used some of Richard's chemicals, and it is amazing what they can do. And finally, Emily.

Emily Anne Bates: My name is Emily Bates, and I work at the University of Colorado in the Anschutz Medical Campus. I am in the Department of Pediatrics. I have been studying bioelectricity since about 10 years ago, when I started my first assistant professor position.

I study how ion channels impact developmental signaling. I started with working on Kir2.1 and inwardly rectifying potassium channels, and now I am kind of branching out and doing screens in Drosophila for all different kinds of ion channels that affect developmental signaling. 


\section{Life as a Bioelectricity Researcher and an Editor}

DSA: Thank you, everybody. I would like to go back to Mustafa. You were the very first person who said "yes" to being an associate editor on the journal, and I know you are an incredibly busy person, and yet you were right there, I think it was within hours of when I sent out the e-mails.

Why did you agree despite how much you have to do, and what is your view of what our journal, Bioelectricity, will become over the next couple of years and in the long term?

MD: Wow. That is a very, very good question. Before I answer your question, which presses on a very sensitive nerve, I just want to say, because a couple of colleagues had touched on somewhat historic aspects, my interest in bioelectricity started as a teenager, when I built a radio transmitter. And I got electric shocks about 50 times a day while doing this. And I was absolutely fascinated with what my body was doing with this shock. And the rest is history. It became a career. With that said, I do not advise that you go around poking your fingers in electric sockets and things.

Dany, the answer to your question is this: in the '90s, when I became curious about the electricity of cancer cells, it was pure curiosity. My father happened to die of cancer at a time when people in Cyprus did not even know what cancer was. I started questioning whether cancer cells generated electrical signals, and more importantly, whether aggressive cancers differed in their electrical signaling than normal cells and nonmetastatic cells and so on. So we started the patch clamping, discovered the voltage-gated sodium channel, and so on and so forth.

When I started then the business of publishing this work, the response was incredible - as in, incredibly bad. The ion channel people did not hesitate much, but the medical profession thought, you know, "This man is crazy. This is an epiphenomenon. Ion channels belong to brain and central nervous system and muscles and this and the other."

And then I put up a fight. And when I actually sat down and said, "What am I going to do about this?" I was convinced I was onto something. But the only way is to educate the field.

So I spent 6 to 7 years, through reviews and through seminar presentations and conferences, convincing ultimately the oncologists and the medical profession at large that bioelectricity is important to medicine. And that is the end I come from.

And when the journal idea came about, I mean, I had no hesitation, because I saw the journal as serving a major, major role - if I may say so, for no less than humankind - in putting forward something that Colin McCaig has done through his reviews: Why is bioelectricity important? Why are bioelectrical signals important? And so on.

So this was just it. This was a formalization of what was a timely and important development that had to be had in biology and medicine. I had no hesitation at all.

DSA: Who else has had that experience of trying to get people to pay attention and finding that one group or more than one group is really hesitant to think about it? Mike, I know you have experienced it. Can you address that?

ML: Sure. One interesting thing I find is that different groups have problems with different aspects of the work. I can always tell what kind of department I am giving a talk in based on which part of the talk makes people scowl. For example, certain things you can say to neuroscientists that they find completely obvious, but the same thing in a molecular genetics department is heresy. Bioengineers, cancer biologists, regenerative medicine people, developmental biologists, synthetic biologists, and cognitive science folks all have different ideas of what's obvious, surprising, or impossible.

And publication-wise, it becomes really interesting. I specifically remember the tail regeneration paper [Development, 2007] that Dany and I did together, on the role of channels and pumps in tail regeneration in tadpoles. ${ }^{1}$ We found that tadpoles used a vertebrate proton conductance to establish a specific voltage state required for regeneration, but we could shut it down and replace it with a completely heterologous proton pump from yeast, with no structural or sequence homology, and everything worked as before, because the instructive physiological state was right.

One of the reviewers was a molecular biologist, and their opinion was, 'Ah, you have found this channel, which is the gene for tail regeneration, so get rid of all this talk about voltage and just talk about the gene for tail regeneration."

And of course, the other reviewer was a physiologist, and they said, "Well, you have shown you can replace that channel with a completely different pump and it still works, so forget all the genetics, don't focus on the channel, and just leave all the physiology." And the editor said, "Yes, very good. Now do both of those things, and also make it shorter" "So we had a heck of a time!

And this comes up again and again, because it is actually quite difficult to find reviewers with appropriate expertise that agree on anything. I think it is getting better now that the audience and the community is growing. But trying to get reviewers who see the big picture beyond the individual silos has definitely been a challenge. Still, I think it is absolutely getting better. My hope for this journal is that it will serve as a beacon for this field, where we will manage the review process to address this problem and provide refereeing that is high-quality and well-informed in this field in order to drive the field forward.

DSA: I like your choice of words there: this journal will be a beacon. Min, you have also been in the field a long time, and you said you started in trauma surgery. That is an interesting leap, and I wonder if you have a different perspective from having been in medicine and now trying to convince physicians that this is important. Can you add to this conversation?

MZ: Of course. I come from a different perspective. In medicine, for the physician ... the patients, they probably are less worried about the mechanism or real science behind what makes something work. If it works, it works. For example, if you look at wound healing, if the wound heals, that's the goal, no matter what approach you took. If it heals, it's good.

One of the critical things with respect to wound healing, however, is to make the cells migrate into the wound or grow into the wound, which is the healing process. If the cells do not go there, then the wound does not heal.

When I was a graduate student in late 1980s, I had a long conversation on healing of tissue damages with my supervisor professor Zhengguo Wang, a founding father of modern trauma surgery in China. He happened to read something about naturally occurring electric fields at wounds, and that kept me super interested in this direction. 
I agree with what Mike just said. When you talk to different people about bioelectricity, the responses can be totally different

I think this journal, Bioelectricity, is especially important. The journal will introduce the field of bioelectricity to multiple audiences and get their attention and understanding of what we are doing and what is happening in this field.

\section{The Education of a Bioelectricity Researcher}

DSA: I think that is a really important point and it is one of our main goals: to keep this journal open and welcoming and educational-and to spread the word and grow the field.

Ann, I know you think a lot about not just bringing in young scientists, but really bringing all scientists into this field to understand the connections. Could you add your perspective on that?

AR: Yes and yes. One of the things that will come out of Bioelectricity, I think, and that is a strength of this whole area - and it has been alluded to already - is how multidisciplinary bioelectricity is. It requires skills and talents from molecular biology and ion channel physiology and electrophysiology and all kinds of things.

The problem is, when people review our papers, they sometimes know something about one aspect of it, but they do not understand all aspects of it. We need to develop a body of people who understand how they knit together to form this new field of bioelectricity. And I think that will be a strength of Bioelectricity.

For example, when we think back to difficulties that we have had in trying to get papers published, as Mike was alluding to earlier, my most memorable review of a grant once by a referee was one sentence, and it said, "Does anyone really believe this stuff anymore?" And that was it. And that was the entirety of their feeling about the credibility of this work. And of course, they are completely wrong.

\section{DSA: Completely! This journal will put those doubts to rest.}

AR: We will also show people how multidisciplinary we are: we will take those aspects of the "proper" science that everyone believes in and put them together in a way that proves, "this is real stuff."

Another example: You take people who understand fully the concept of a membrane potential, and there is no problem with that, but then you introduce the concept of a transepithelial potential, where you have a voltage difference across a tightly sealed epithelium, and for some reason, this is a huge leap of faith for them, and I have never quite understood the difficulty in this concept.

But if you break it down and explain it to people and tell them about the physiology behind it, suddenly you can almost see a light switch on, and then they understand. Bioelectricity will take people through the complexities of it piece by piece and introduce people to how it fits together to make a much bigger picture.

Our students are really good at this. We take students as undergraduates - and I am involved in teaching on a variety of topics, including neural development and regeneration and physiology, cell biology—and you can introduce concepts into individual courses.

By the time they come to their final year, you can put it together for them in a way that makes a lot of sense. And again, you can see the light switch on, and you can see how enthused they are about it. And we have no trouble getting students to volunteer to work in our labs on lab-based honors projects, because they become really excited about it, as excited as we are about it. I think that is the future, and Bioelectricity will play an important role.

DSA: I am very pleased to hear that-very pleased. I want to take this now to Emily. Can you tell us a little bit about your educational experience? Could you tell us what exactly caught you. When did the light go on for you?

EAB: It was just in learning about the channelopathies, and that these mutations occurred in ion channels. I had done my whole undergraduate research training for 4 years in a developmental biology lab as an undergraduate, and I had taken upper-level developmental biology classes. I knew that I had learned about all of the canonical developmental signaling pathways, and none of them had mentioned ion channels.

My plan was to work on neurological disease, so I learned about ion channels and neuroscience as a $\mathrm{PhD}$ student. No one mentioned the role of ion channels in development. So there seemed to be two totally separate fields.

In my postdoctoral work in Louis Ptacek's lab, I saw that channelopathy patients had a syndrome of birth defects. Then I learned about some of the other channelopathies, like Timothy syndrome. The developmental symptoms were similar among channelopathy syndromes. How ion channels could affect development felt like a mystery to me.

When I started, it was hard to put the bioelectricity field together, coming into it from just a mystery aspect. I just started doing the science. As we learned more from our experiments, it became more and more interesting to me, that these ion channels could influence the canonical developmental signaling pathways. It just seemed like the fields of development and ion channel biology had been very separate.

I think the difficulty in following the field and bringing the research together is one of the reasons we really need this journal, Bioelectricity. We need to start having more conferences that bring us together to allow us to have a bioelectricity community to discuss our questions and our findings. I agreed to be an associate editor because I feel like the journal is one of the field's biggest needs right now. I would have loved to have this journal when I started! And also, I agree with what Ann said-it is great to be able to talk about this field with undergraduates and with graduate students, and at conferences, as most of us do, I think, to spread the word about this really exciting field.

DSA: I gather you put this together for yourself, and I think that is the experience of many of us. Does anybody else want to comment on that?

RK: Sure. I'll chime in. I am a little bit of an outlier here in that I have never called myself a bioelectricity researcher. I have just studied electrophysiology and channels and 
electrical signaling, and sometimes they have been in neurons and sometimes not.

I have been aware for my entire career about the role in electrophysiology in development and regeneration, et cetera. So it is a little surprising, as someone who is a bit of an outsider, to hear the resistance that you guys have encountered to this idea, because the importance of ion channels to just about everything seems so obvious and just essential to what I have learned throughout my education.

\section{Tools for Bioelectricity}

DSA: Richard N., I want to segue into talking about medicine. One of the main topics that will be covered in the journal is technology: tools for studying bioelectricity, and how tools we use can be useful to physicians. And that is what you work on.

Could you talk a little bit about your work and how you think having a section devoted to technological advances can contribute to the field and the journal?

RN: Certainly. Since the electric field is one of the fundamental forces of nature, it can be used as a tool to modify the state of cells through signaling. The application of steady, direct current electric fields has been shown to influence cell migration and wound healing and pulsed electric fields can penetrate into cells and organelles to directly influence many different cellular functions, such as secretion, mitosis, and programmed cell death. It is this latter application that is particularly useful to oncologists faced with treating various forms of cancer. We find that applying the appropriate number of 200-ns-long pulses triggers immunogenic cell death in every type of tumor studied. Not only does the tumor self destruct, but it alerts the immune system of its presence to generate cytotoxic T cells that search and destroy other tumor cells. This is a nonthermal, drug-free therapy that only takes a few minutes to trigger a cell death pathway that is integral to all cells. Clearly there will be much interest in using bioelectric tools such as this to treat various disease states.

DSA: The tools we publish will be very important, I agree. Richard K., can tell us a little bit about your tool and how it is different?

RK: Okay. There are tools-the sort of things that we have made-which are chemical actuators or manipulators of either channels, or receptors, or more generally, membrane voltage. There is also a whole new generation of voltage indicators, voltage-sensitive dyes, and genetically encoded voltage sensors that are emerging. A close colleague of mine at Berkeley, Evan Miller, is about to make a big splash with a new voltage-sensitive dye, which, by the way, he is primarily using on nonneural cells.

The venues for publishing basic cellular electrophysiology have been changing over the years. The high impact neuroscience journals, including Neuron and Nature Neuroscience, have a very big task in covering the whole spectrum of research in the nervous system. And the emergence of new techniques has resulted in many more papers focused on circuits, connectomics, on "big science" sorts of things, and relatively less on cell or molecular physiology.
So, people have been scrambling to try to find good journals to publish good papers in these fields. So I think that there is a big need for a fresh new venue that publishes papers about new technology, new mechanisms, and new physiological outcomes relevant for bioelectricity. It's very competitive and somewhat arbitrary what sort of studies make it into the top tier journals, and there are potentially a lot of people confused about where next to submit their otherwise excellent paper. If Bioelectricity is known to be an inclusive journal, which I would argue it is, it will really do well and capture a lot of interesting papers from many fields, including advances in technology.

\section{Bioelectricity in Medicine}

DSA: Annarosa, you have been studying ion in channels in cancer for many years now. In fact, we first met at an Ion Channels in Cancer meeting you organized. Would you tell us about the reception your ideas have received in the medical community?

AA: One of the main problems I encountered during my scientific life, was to convince people that the hERG1 channels could be considered good targets in oncology, despite the fact that they are commonly identified as "undesirable" targets. I think (and I have got good feedback from the "pharma" world) that novel therapies, based on targeting ion channels, including hERG1, will be considered for cancer treatment in the near future.

DSA: Mustafa, you also work on cancer. In addition, you have also been looking at side effects of things like anesthesia that affect ion channels.

I am curious as to how the medical community has reacted to that, as well as the work you have done on ion channels in cancer. For example, has that work been accepted? Are people excited to have a hypothesis about these side effects? Is that something that people are listening to and welcoming?

MD: Indeed. I think we have not done justice to what you call side effects. In fact, what we are talking about is the fact that anesthesia and cancer, what I work on, are connected, obviously. But they are also connected through ion channels. So you could actually-and there is evidence for it-affect cancer progression through anesthesia, or medication like painkillers, some of which also affect ion channels.

DSA: You bring up something else very important that Bioelectricity will impact in important ways. That is that there are many drugs that target ion channels and pumps and are already approved for use in humans. Some of them are even over the counter. I, and I am sure many of you, have argued that this makes these "electropharmaceuticals" exciting candidates for use in the myriad situations, like cancer, that we are discovering can be treated by controlling ion flux. Bioelectricity will be the leader in bringing these off-label uses to light.

Min, you also have something that is going into clinical trials. Do you want to discuss how our journal could be used to foster useful interaction between medicine and bioelectricity researchers? 
MZ: I think having a "tools" section is key, and I know we are going to include that section in the journal. For example, Lionel Jaffe and Richard Nuccitelli's work on vibrating probes [self-referencing ion-selective probes] has been, well, in this field for over half a century. We do not really have newer ones that have similar function. We are still using it. Bioelectricity is where we will turn to find out new technology.

For the clinical part, I am not quite sure, because a lot of people in clinical practice are using electric stimulation that are not necessarily very scientific. The meaning attached to bioelectricity ... as Ann said before, people think, "Well, this is a field and there are a lot of bad things attached to it. Why are those people still working on it?" We must work to avoid that kind of impression and get out of that mode of thinking. I will be glad to see newer technologies introduced and will encourage scientists to submit that kind of research to the journal.

\section{Frankenstein Was Not a Bioelectricity Researcher}

AR: I absolutely agree. As we both will have experienced in the past, and many of us will have experienced, people still see ... in their heads they conjure up an image of Frankenstein.

MZ: Yes.

AR: And we are not doing Frankenstein science.

DSA: A quick side note: In Shelley's original story, Frankenstein was not doing science at all. Behind the backs of the scientists who are his graduate advisors, he is practicing alchemy. That is certainly not what bioelectricity is.

AR: Absolutely! There is real, genuine physiology and biophysics behind what we do, and we will educate people to the understanding that there is real science here. We are not doing galvanic reanimation here. That is not what we are about. We are about real science.

I think we have all found that as we have published papers and put in our grant applications, we have had to be a little bit more careful and had to have extra work, and maybe a mechanism that would not have been necessary for the first publication of a phenomenology in a different subject area. So I think we have often felt that we have had extra rigor demanded of us by the refereeing community. And so we have evolved into a community of scientists that are very careful, and I think we should be very proud of that, and that will come through in our journal.

DSA: That is an interesting observation, and I had not thought of it that way. But you are right. There is a level of care and robustness in our results that is something we should be proud of and we should highlight.

\section{Bioelectricity and Bioelectricity}

DSA: We've been talking about some specific categories of research we will publish: basic research, medicine, creating new tools. Let's zoom back out again and talk a little about the big picture. What is your vision for the journal?

RK: One question in terms of the journal is, how broadly are we defining bioelectricity? Of course, we have a community of people that already want to publish here. Do you see it growing to include a wider range of the role that electrical signaling plays in excitable and nonexcitable cells? Cancer, immune responses, endocrine and exocrine function-there are an endless range of interesting processes involving or influenced by electrical events.

What are we going to use as our definition of bioelectricity?

DSA: That is a great question. I am just going to jump in here. At the beginning, I thought, "Well, this is going to be anything that does not go to a neuroscience journal or a biophysics journal." So, nonexcitable cells-although I agree that excitable versus nonexcitable is maybe a specious distinction. But of course it cannot be defined by what it is not.

MD: I think that it is the fundamental question which I presume, Dany, you will tackle in your first editorial.

DSA: Yes. And probably in many editorials over the years. From the very beginning, though, this group of people that is on this phone call right now-you, the associate editorswill work together to make sure this field has a strong central foundation on which to build. Richard, in answer to your question, we will publish subject overviews, perspectives, and book reviews. That will include work done by the pioneers talking here today, including work on development, regeneration, cancer, tools, medical advances, wound healing, etc. Alongside that, we will publish the best work from the "endless range of interesting processes," such as work on bioelectricity in microbiology, botany, immunity, etc. We need to tend to the field as it grows while letting it grow according to the curiosity of all interested scientists.

EAB: I also think we should err on the side of inclusion, because we want the field to grow, and people can learn more about it by becoming familiar with the journal. I have learned a lot from the neuroscience community and also from the development community.

While I want there to be a journal where we have a collection of reviewers and editors that understand the aspects of ion channels and the aspects of development and regeneration, it is also important that we include more people from other disciplines who start thinking of themselves as associated with the field of bioelectricity.

RN: My feeling is that Bioelectricity should include any research into the interaction with or use of electricity by living things. This would be the broadest definition of our focus but one that will serve to illustrate the relevance of this field to everything that we do.

MZ: I agree with what all has been just said, I also want to see this field as inclusive.

DSA: I agree completely. Mike, like others here, you have done a lot of work as an ambassador for the field. Can you comment on inclusion and distinction?

ML: I think what Emily said is right on. And this ties into a question that I hear all the time, which is, "How many people are actually in this field?" People say, "Well, I have never heard of bioelectricity. How big is the field?' And I think if 
you define it as the people who explicitly call themselves workers in bioelectricity, then it is quite small-very small.

But on the other hand, I think nowadays especially there are lots of people that work in bioelectricity that do not necessarily know they work in bioelectricity. For example, there are lots of people who work on various channelopathies from a genetics angle: They eventually clone the gene and find that now they have to think about how ion channels regulate patterning.

I sometimes see these channelopathy papers for example, and I will e-mail the authors, and say, "Hey, did you know all this other stuff existed?" And they say, "Oh, my God, incredible. We have never heard of any of this, but it sure helps us understand what's going on." Same with bioengineers who apply electric fields not knowing about the endogenous roles, cancer biologists who pull out ion channels as oncogenes, or neuroscientists who don't think about the fact that many cells can actually do what neurons do (just slower), and had been doing it for a long time before brains came on the scene. Also, for example all the people in synthetic biology that are making interesting new materials that are absolutely relevant for us, all of these people may or may not know they are in the field, but I think they are in the field.

And I agree with Emily that I think our goal is to bring them into the fold and help forge the connections, because I think that is one of the most interesting aspects of this field: it is not just another set of biophysical mechanisms that developmental biologists now have to worry about, but rather, there are some amazing conceptual aspects of bioelectricity that may link this work to all kinds of areas in synthetic biology and origin of multicellularity and primitive cognition and even aspects of things like unconventional computation.

And so I think we should absolutely err on the side of pulling everybody in, and then seeing what the center of gravity ends up looking like and where the borders of this discipline might turn out to be.

EAB: I would just like to say that is exactly how I was brought in. Mike mentioned e-mailing people that publish on channelopathies. Mike sent an e-mail and sent me a few papers, and that is how I met him and then, through him, I met Dany and others. So I am grateful to Mike that he reaches out like he does.

AR: I just want to say I agree with what Emily and Mike and you as well, Dany, have said. We should be inclusive, because we do not have a crystal ball. We cannot predict what technologies and techniques might emerge in the future. So we need to keep an open mind about this. As much as we would expect other people to have open minds for us, we should have one for ourselves, as well.

So I think one of the things that Mike mentioned in particular was this thinking about bioengineering and new materials and new techniques. We cannot see into the future and know for sure what or how this work that we are doing that relates to developmental biology might end up leading to a clinical situation that could be aided using bioelectrical phenomena. And that is the sort of thing that Mustafa was mentioning in the introduction.

I think the whole thing can go full circle-what we do in the petri dish, what we learned from embryos-and then transferring that into the clinic. And new materials, new engineering possibilities are just emerging every day. We should just keep an open mind about it.

\section{DSA: Richard, are we answering your question?}

RK: Yes. For example, someone working on synapse development, that is clearly neuroscience, but it is part of development and there have been suggestions about electrical fields driving receptors to aggregate electrophoretically. There is a lot of overlap, I think.

And I guess I would be hesitant to define this field by "what it is not" - you know, saying it does not necessarily include the nervous system or conventional synapses.

DSA: Correct, we cannot say "these are the boundaries," at this point, because, as you all have been saying, you cannot predict who is going to come up with the next imaginative, wonderful combination that will appear on our doorstep, and we should be able to say, "Yes, let's include this." What we can do is say, "This is the field, so far."

\section{RK: Right.}

This is what Ann was saying earlier. Bioelectricity, by definition, is multidisciplinary. We are all coming at many questions from different angles. In short, there is a lot to cover, and we have a lot to do. But this journal is an exciting development, and I think that we all share in the excitement.

\section{DSA: We are at a very exciting moment indeed.}

Bioelectricity will provide a focal point for a dynamic field, a task that will require many skills. Our editorial decisions will inevitably influence who decides to calls themself a bioelectricity researcher. I take that responsibility very seriously, and I know you do as well.

\section{Last Words}

DSA: We've talked about the role of the journal as an anchoring point for an inclusive and diverse field, and the importance of having a cadre of people who can review these kinds of papers. We've also discussed how our own experiences have informed our view of the journal's role in discovery and in education, and how the journal will spread the word to the scientific community in general. Finally, I very much appreciate your thoughts and insights into the importance of being inclusive and open to different research questions, novel ideas, and inventive tools for research and medicine.

Would anybody like to add some final thoughts?

RN: Bioelectricity encompasses all of life, and life would not exist without it. That this fact is not common knowledge serves as a challenge to all of us. We must not only publish the most exciting new findings, but we must publicize them to the nonscientists out there so that everyone can appreciate the marvels of bioelectricity.

ML: Bioelectricity is an incredibly exciting emerging field: it's a perfect example of an interdisciplinary undertaking that spans from basic science (evolutionary developmental biology) to biomedicine (regeneration, cancer, and birth defects). It is an ideal place for energetic young scientists to look for the next open vistas of discovery for their careers. We need to make this journal a central touchstone for existing workers and bioelectricity researchers-to-be-the place to go 
to see the latest discoveries, and the place to send their work for the best review experience.

AA: We all are convinced that bioelectricity is an emerging field, spanning from basic science to medicine. Our main job, through our journal, will be to join bioelectricity researchers to the whole scientific community, to broaden this relevant scientific field.

MD: I just want to say, finally, as possibly the oldest person around the table, that in my experience of having done various editorial things that the single most important factor to sell the journal is the editor, editor, and editor.

So I think, Dany, that is obviously you, but you have got the associate editors. I think you should use the associates very well, and altogether, to make a success of what joins us together today, and that is the success of the journal and the field at large.

DSA: That's another important point. As we've been discussing, the field is multidisciplinary and has insights and tools to offer to a wide range of other fields as well as other scientists and everyone else. We are all invested in the success of this endeavor. And thank goodness, because no one could do this alone. So, I will take your comment, Mustafa, as an offer and point out that it is an honor to be part of this team. To say $I$ have faith in you all is ridiculous-zero faith is necessary. There is so much evidence of the excellence and enthusiasm of each of you, and it is clear from this roundtable that, as a team, we will reach our goals for this journal and this new field.

Thank you all very much for your time today and in the future.

\section{Author Disclosure Statement}

No competing financial interests exist.

\section{Reference}

1. Adams DS, Masi A, Levin M. H+ pump-dependent changes in membrane voltage are an early mechanism necessary and sufficient to induce Xenopus tail regeneration. Development 2007;134:1323-1335. 